\title{
CONVERGENCE-PRESERVATION CRITERIA FOR A GENERALIZED HAUSDORFF MEAN
}

\author{
C. W. LEININGER
}

1. Introduction. The generalized Hausdorff method denoted by $H^{(s)}(d)$ is defined in [4] to the effect that if

(1) $s$ is a sequence of positive numbers,

(2) $d_{n}=\int_{[0,1]} I^{n} d g, n=0,1,2, \cdots$, and

(3) where $n, p$ is a nonnegative integer pair,

$$
\left(\begin{array}{l}
n \\
p
\end{array}\right)_{8}
$$

denotes 0 if $n<p, 1$ if $n=p$, and $s_{n} \cdot s_{n-1} \cdots s_{p+1} /(n-p) !$ if $n>p$, then

$$
H_{n p}^{(s)}=\left(\begin{array}{l}
n \\
p
\end{array}\right)_{s} \Delta^{n-p} d_{p} .
$$

If $s_{n}=n, n=1,2,3, \cdots$, then $H^{(s)}$ is denoted by $H$ and $H(d)$ represents the Hausdorff method. Furthermore $H^{(s)}$ is expressible in terms of $H$ by the formula $H_{n p}^{(s)}=\pi_{n}^{(p)} H_{n p}$, where $\pi_{p}^{(p)}=1$ and $\pi_{n}^{(p)}=\prod_{k=p+1}^{n} s_{k} k, n>p$. As in [4] $s$ is restricted so that $s_{n} \leqq n, n$ $=1,2,3, \cdots$.

Let $R$ denote the space of sequences $d$ each of which is generated by a function $g$ which is Riemann-integrable on $[0,1]$, and let $B V$ denote the subspace of $R$ such that $d \in B V$ if $g$ is of bounded variation on $[0,1] . H^{(s)}(d)$ means generated by sequences of the latter type were considered in [4]. In this paper we are primarily interested in moment sequences belonging to $R$ but not to $B V$. It is found that the three conditions of Silverman and Toeplitz are reduced to two, and a generalization is obtained of one of the fundamental theorems of $H(d)$-summability. Conditions on $s$ and $g$ under which $H^{(s)}(d)$ is multiplicative are established and an example is indicated to show that the restriction on $g$ is not necessary.

2. Modification of the Silverman-Toeplitz conditions. If $d \in R \backslash B V$, the three conditions for convergence-preservation reduce to two in the case of an $H^{(s)}(d)$ mean, namely, that $\left\|H^{(s)}\right\|$ exists and $\left\{\sum_{p=0}^{n} H_{n p}^{(s)}\right\}$ converges. Before establishing this we have, with $c_{0}$ denoting the space of zero-limit sequences, the following property of the collection of product sequences.

Presented to the Society, April 12,1968; received by the editors March 6, 1967 and, in revised form, September 29, 1967. 
Lemмa 1. If there is a nonnegative integer $P$ such that $\pi^{(P)} \in c_{0}$, then for each nonnegative integer $p, \pi^{(p)} \in c_{0}$.

Proof. If $p<P$ and $n \geqq 1, \pi_{n}^{(p)} \leqq \pi_{n}^{(P)}$. If $\epsilon>0$ and $p>P$, then there is a positive integer $N$ such that if $n>N, \pi_{n}^{(P)}<\epsilon \prod_{k=P+1}^{p} s_{k} / k$, so that $\pi_{n}^{(p)}=\pi_{n}^{(P)} / \prod_{k=P+1}^{p} s_{k} / k<\epsilon$.

We next show that if $d \in R \backslash B V$ and $H^{(s)}(d)$ is conservative, then it is multiplicative.

THEOREM 1. If $d \in R \backslash B V$ and there is a number $K$ such that $\sum_{p=0}^{n}\left|H_{n p}^{(s)}\right|<K, n=0,1,2, \cdots$, then $\lim _{n} H_{n p}^{(s)}=0, p=0,1$, $2, \cdots$.

Proof. Since $d \notin B V$, the sequence $\left\{\sum_{p=0}^{n}\left|H_{n p}\right|\right\}$ is unbounded. Suppose there is a nonnegative integer $P$ such that $\pi^{(P)} \notin c_{0}$. From Lemma 1 if $p \geqq 0$, there is a positive number $t_{p}$ such that $\lim \pi^{(p)}=t_{p}$. Furthermore $t_{0} \leqq t_{p}$ so that if $n \geqq p, \pi_{n}^{(p)} \geqq \pi_{n}^{(0)} \geqq t_{0}$ and $\sum_{p=0}^{n}\left|H_{n p}^{(s)}\right|$ $\geqq t_{0} \sum_{p=0}^{n}\left|H_{n p}\right|$. Therefore $\pi^{(p)} \in c_{0}$ if $p \geqq 0$, and since the set $\left\{H_{n p}\right\}$ is bounded, $\lim _{n} H_{n p}^{(s)}=0$.

It may be observed that if $d \in R \backslash B V$, then the existence of $\left\|H^{(s)}\right\|$ is necessary and sufficient that $H^{(8)}(d)$ be regular over the space $c_{0}$ [2, p. 49, Theorem 4].

There remains only the statement of conditions necessary and sufficient for convergence-preservation.

ThEOREM 2. If $d \in R \backslash B V$, then $H^{(s)}(d)$ is multiplicative if and only if there exist numbers $K$ and $L$ such that

(i) $\sum_{p=0}^{n}\left|H_{n p}^{(s)}\right|<K, n=0,1,2, \cdots$;

(ii) $\lim _{n} \sum_{p=0}^{n} H_{n p}^{(s)}=L$.

3. A fundamental theorem. If $a_{n}=1-s_{n} / n, n=1,2,3, \cdots$, it is apparent from the proof of Theorem 1 that if $d \in R \backslash B V$ and $H^{(s)}(d)$ is conservative, then $\sum_{n} a_{n}$ is divergent. Hence in view of $[4$, Theorem 6] we may extend one of the fundamental theorems of Hausdorff summability.

TheOREM 3. If $\sum_{n} a_{n}$ is convergent, then $H^{(s)}(d)$ is conservative if and only if $d \in B V$.

4. Sufficient conditions for convergence-preservation. For each nonnegative integer pair $n, p, n \geqq p$, let $f_{n p}$ denote the polynomial

$$
\left(\begin{array}{l}
n \\
p
\end{array}\right) I^{p}(1-I)^{n-p}
$$


and $V_{[a, b]} f_{n p}$ the variation of $f_{n p}$ on $[a, b]$. Then $H_{n p}=\int_{[0,1]} f_{n p} d g$. We state some lemmas for convenience.

LemMa 2. If $n$, $p$ is a positive integer pair, $n>p$, then

(i) $f_{n p}$ is increasing on $[0, p / n]$ and decreasing on $[p / n, 1]$;

(ii) $V_{[0,1]} f_{n p}=2 f_{n p}(p / n)$;

(iii) $V_{[0,1]} f_{n, n-p}=V_{[0,1]} f_{n p}$;

(iv) $\lim _{n} V_{[0,1]} f_{n p}=2 p^{p} e^{-p} / p$ !.

Lemma $3 . \lim _{p} p^{p} e^{-p} / p !=0$.

Lemma 4. If $d \in R$ and $\epsilon>0$, there is a positive integer pair $N, P$ such that if $n>N$ and $P \leqq p \leqq n-P$, then $\left|H_{n p}\right|<\epsilon$.

Lemma 5. If $0<t<1$ and $p$ is a positive integer, then $\lim _{n} V_{[0, t]} f_{n, n-p}$ $=0$.

Lemma 2 may be established by computing $f_{n p}^{\prime}$ and noting that the high point of $f_{n p}$ is at $p / n$, and Lemma 3 follows from an application of Stirling's formula. Lemma 5 is readily obtained from Lemma 2 . Since the author is not aware of the existence in the literature of a proof of Lemma 4, an argument therefor is given after the theorem.

THEOREM 4. If $d \in R \backslash B V$ and there is a number $M$ such that $\sum_{p=0}^{n} \pi_{n}^{(p)}<M$, then $H^{(s)}(d)$ is regular over $c_{0}$. Furthermore if $g(1-)$ exists, $H^{(s)}(d)$ is multiplicative, and if $g(1)-g(1-)=2, H^{(s)}$ (d) is regular.

Proof. If $W$ is a number such that $\left|H_{n p}\right|<W, n \geqq p, p=0,1$, $2, \cdots$, then $\sum_{p=0}^{n}\left|H_{n p}^{(s)}\right|=\sum_{p=0}^{n} \pi_{n}^{(p)}\left|H_{n p}\right|<M W$, and $H^{(s)}(d)$ is regular over $c_{0}$.

If $\epsilon>0$, from Lemma 4 there is a positive integer pair $N_{1}, P$ such that if $n>N_{1}, \sum_{p=P}^{n-P}\left|H_{n p}^{(s)}\right|=\sum_{p=P}^{n-P} \pi_{n}^{(p)}\left|H_{n p}\right|<\epsilon / 3$.

From Theorem 1 there is a positive integer $N_{2}, N_{2} \geqq N_{1}$, such that if $n>N_{2}$, then $\sum_{p=0}^{P-1}\left|H_{n p}^{(s)}\right|<\epsilon / 3$.

From Lemma 2(iv) there is a number $V$ such that if $0<p<P$ and $n>p$, then $V_{[0,1]} f_{n p}<V$. Hence by Lemma 2(iii), if $n-P<n-p<n$, then $V_{[0,1]} f_{n, n-p}<V$. If $\epsilon>0$, there is a positive number $t$ such that if $t \leqq x<1$, then $|g(x)-g(1-)|<\epsilon / 9 P V$. Let $U$ denote an upper bound on $[0,1]$ for $|g|$. From Lemma 5 there is a positive integer $N, N \geqq N_{2}$, such that if $n>N$ and $0<p<P$, then $V_{[0, t]} f_{n, n-p}<\epsilon / 18 P U$. Since from Lemma 2 (i) $f_{n, n-p}$ is decreasing on $[(n-p) / n, 1]$, for each $n$ there is a number $z_{n}, t<z_{n}<1$, such that $V_{\left[z_{n}, 1\right]} f_{n, n-p}<\epsilon / 18 P U$. Thus if $n>N$, 


$$
\begin{aligned}
& \left|H_{n, n-p}^{(s)}\right| \leqq\left|\int_{[0,1]} f_{n, n-p} d g\right| \leqq\left|\int_{[0, t]}[g-g(1-)] d f_{n, n-p}\right| \\
& +\left|\int_{\left[z_{n}, 1\right]}[g-g(1-)] d f_{n, n-p}\right|+\left|\int_{\left[t, z_{n}\right]}[g-g(1-)] d f_{n, n-p}\right| \\
& <2 U V_{[0, t]} f_{n, n-p}+(\epsilon / 9 P V) V_{\left[t, z_{n}\right]} f_{n, n-p}+2 U V_{\left[z_{n}, 1\right]} f_{n, n-p}<\epsilon / 3 P .
\end{aligned}
$$

Therefore if $n>N$, then $\sum_{p=0}^{n-1}\left|H_{n p}^{(s)}\right|<\epsilon$, and it is well known (e.g., see $[3])$ that $\lim d=[g(1)-g(1-)] / 2$.

An example of a function $g$ showing that the existence of $g(1-)$ is not necessary for convergence-preservation may be constructed by defining $g(x)=h(1-x)$ where $h$ is defined in $[1$, p. 119, Theorem 3$]$ and observing that $f_{n, n-p}(x)=f_{n p}(1-x), 0 \leqq x \leqq 1$.

5. Proof of Lemma 4. For the convenience of the reader the lemma is restated.

Lemma 4. If $d \in R$ and $\epsilon>0$, there is a positive integer pair $N, P$ such that if $n>N$ and $P \leqq p \leqq n-P$, then $\left|H_{n p}\right|<\epsilon$.

Proof. If $\epsilon>0$, then from Lemmas 2 (iv) and 3 there is a positive integer pair $N_{P}, P$ such that if $n>N_{P}$, then $V_{[0,1]} f_{n P}<\epsilon / U$, where $|g|<U$ on $[0,1]$.

We next show that if $n$, $p$ is a positive integer pair, $p \leqq n / 2$, and $p \leqq q \leqq n-p$, then $V_{[0,1]} f_{n q} \leqq V_{[0,1]} f_{n p}$. If $p=n / 2$, then $q=p$. If $n=3$, then $q=p$ or $q=n-p$, and the conclusion follows from Lemma 2(iii). Suppose there is a least positive integer, denoted by $k+1$, such that if $p<(k+1) / 2$, then there is a positive integer $q$ such that $p \leqq q \leqq k$ $+1-p$ and $V_{[0,1]} f_{k+1, q}>V_{[0,1]} f_{k+1, p}$. From Lemma 2 (iii) $q<k+1-p$. Furthermore if $p \leqq q \leqq k-p, \quad V_{[0,1]} f_{k q} \leqq V_{[0,1]} f_{k p}$. Since the sequence $\left\{(1+1 / n)^{n}\right\} \quad$ is increasing, $\quad(k /[k+1])^{k}(1+1 /[k-q])^{k-q} V_{[0,1]} f_{k q}$ $\leqq(k /[k+1])^{k}(1+1 /[k-p])^{k-p} V_{[0,1]} f_{k p}$, whence using Lemma 2(ii), $V_{[0,1]} f_{k+1, q} \leqq V_{[0,1]} f_{k+1, p}$.

Thus if $N=N_{P}+2 P$ and $n>N$, then $\left|\int_{[0,1]} f_{n p} d g\right|=\left|\int_{[0,1]} g d f_{n p}\right|<\epsilon$.

\section{REFERENCES}

1. J. P. Brannen, Concerning Hausdorff matrices and absolutely convergent sequences, Proc. Amer. Math. Soc. 15 (1964), 114-123.

2. G. H. Hardy, Divergent series, Clarendon Press, Oxford, 1956.

3. R. E. Lane, The integral of a function with respect to a function, Proc. Amer. Math. Soc. 5 (1954), 69-76.

4. C. W. Leininger, Some properties of a generalized Hausdorff mean, Proc. Amer. Math. Soc. 20 (1969), 88-96.

UNIVERSITY OF DALLAS 